\title{
Photoelectric-helicoidal spirometer
}

\author{
LUIS CARRETIÉ, JAIME IGLESIAS, and PEDRO AGUILAR \\ Universidad Autónoma de Madrid, Madrid, Spain
}

\begin{abstract}
This paper describes a photoelectric-helicoidal spirometer that has been designed in our laboratory. It includes a cylindrical helix (or turbine) partitioned in each of its blades by means of opaque plates transversally disposed. These plates obstruct the ray of light emitted by a diode, preventing it from reaching a photoreceptor located at the opposite side of the turbine. These basic elements are located in a module that can be adjusted to a mask that covers nostrils and mouth. Control software for this device is also described. The software facilitates a graphical representation of respiratory activity, as well as its storage in the computer. Software has been designed to be adaptable to any digital-to-analog converter. Both the device and the software have several advantages over traditional spirometers, as well as other nonspirometric techniques for studying respiratory activity.
\end{abstract}

Respiration constitutes an important psychophysiological signal that supplies information about very different psychological processes, such as emotion, anxiety, concentration on a task, and so forth (Wientjes, 1992). In part, the interest in this signal resides in the fact that it reflects the activity of the sensorimotor and autonomic nervous systems, since it is controlled by both (Carretié \& Iglesias, 1995; Kaufman \& Schneiderman, 1986). However, despite this interest, it is one of the least studied psychophysiological signals. The principal reason for this relates to technical issues.

All three of the methods most widely employed in the study of respiration involve certain drawbacks that make them not entirely appropriate. Thermistors (which transduce temperature into electricity and consist of small sensors usually placed in the nostrils) detect the temperature changes between the air inhaled and the air exhaled. However, thermistors do not provide information about the absolute respiratory volume and flow (or respiratory slope, a parameter influenced by, among other factors, the diameter of the bronchioles, which is controlled by the autonomic nervous system; see, e.g., Brodal, 1981); they provide information only about the respiratory rate (number of cycles per minute). Furthermore, the recording is not entirely simultaneous with the original respiratory activity (the metal in thermistors involves a certain delay in warming or cooling). Moreover, the recordings strongly depend on the environmental temperature. The recordings tend to present lower amplitudes when the environ-

The photoelectric-helicoidal spirometer is inscribed by the Universidad Autónoma de Madrid in the Spanish Patent Office (11-1-969600046). We are grateful to the company Sistemas-InstalacionesRedes, S.A. (SIR, S.A., Tres Cantos, Madrid) for its aid in relation to the calibration process and determination of the technical specifications of the photoelectric-helicoidal spirometer. Correspondence may be directed to L. Carretié, Dpto. Psicologia Biológica y de la Salud, Facultad de Psicología, Universidad Autónoma de Madrid, 28049 Madrid, Spain (e-mail: carretie( $w$,uam es). mental temperature is high than when it is low (Lorig \& Schwartz, 1990).

The second method is plethysmography, which requires the location of strain gauges or highly sensitive bellows around the body at the level of the thorax or the abdomen. In this case, the biasing variable is the extent to which the gauge, or the bellows, is stretched when it is placed, as this can alter the amplitude of the recordings (Lorig \& Schwartz, 1990). Again, the only accurate information we can extract from traditional plethysmographic recordings (those that employ a single strain gauge) is respiratory rate (Lorig \& Schwartz, 1990; Wientjes, 1992). However, plethysmographic methods that allow for the assessment of respiratory volume and flow, involving the use of pairs of calibrated strain gauges, bellows, or inductive bands, along with multiple regression analyses, have been described (Wientjes, 1992).

Spirometry is the third of the most important methods for measuring respiratory activity. This technique is capable of measuring respiration volume and flux, as well as respiratory rate. In this case, the drawback is the inconvenience involved due to the unmanageability of the instruments. In comparison with the two other techniques described above, spirometers are very large and heavy, since they consist basically of two receptacles containing liquid and air, respectively. The latter is extracted from or injected into the former receptacle during inhalation and exhalation. Moreover, these physical characteristics seriously disrupt the spontaneous breathing pattern (Wientjes, 1992).

This paper presents a spirometer that uses a cylindrical helix (or turbine) instead of tanks to measure the volume of air respired. To simplify both the hardware design and the software programming (the low cost of the device thus being facilitated), the turbine does not move during inspiration. Although the spirometer records only exhalation activity, the capability for measuring the volume and flux of air respired gives this method the advantages of classical spirometers: highly reliable recordings and the 
possibility of measuring parameters apart from respiratory rate. Moreover, the helix-based design allows for a simpler instrumental structure, making its usage more comfortable for both the experimenter and the experimental subject, whose respiratory pattern is less disrupted than it is with classical spirometers, since the subject's head movements are not restricted by air tubes. Finally, as will be explained shortly, the photoelectricity-based detection of helix rotation makes the computer storage of recordings easier.

\section{Description of Hardware}

The spirometer described here, which is schematically represented in Figure 1, is inexpensive, light, and compact. The most important elements are the helix or turbine (1), the light-emitting diode (LED 3), and the lightdetecting diode (LDD 4). Moreover, the turbine is partitioned in each of its blades by means of opaque plates transversally disposed (2). Thus, these plates obstruct the ray of light, preventing it from reaching the LDD, when they interpose themselves between LED and LDD. The number of blades chosen is four, since it is between the minimum limit for effectiveness of the turbine (i.e., it reacts even at low levels of air flux) and the maximum weight limit of the turbine, whose greater weight gives it greater inertia.

This initial structure (partitioned turbine, LED and LDD: Figures $1 \mathrm{a}$ and $\mathrm{lb}$ ) is inserted into a module (5; Figure $1 \mathrm{~b}$ ) open in its anterior and posterior parts in order to allow for the circulation of air. This module is ad-

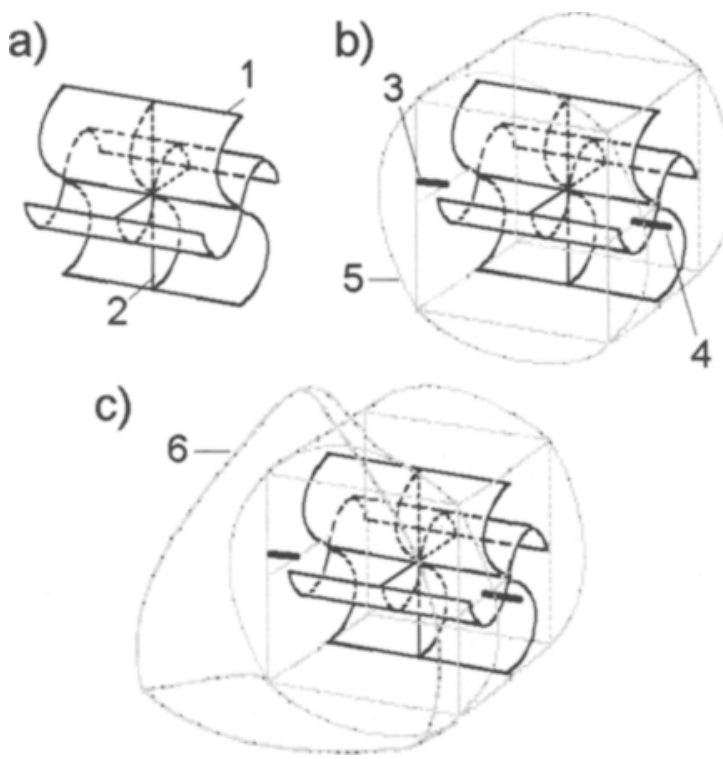

Figure 1. Representation in perspective of the photoelectrichelicoidal spirometer. (a) 1 -turbine, 2 -opaque plates. (b) This initial structure is inserted into a cylindrical module whose interior is an open parallelepiped, which includes the light emitter (LED) and the light detector (LDD): 3-LED, 4-LDD, 5cylindrical module. (c) The module is adjusted to a mask: 6 .

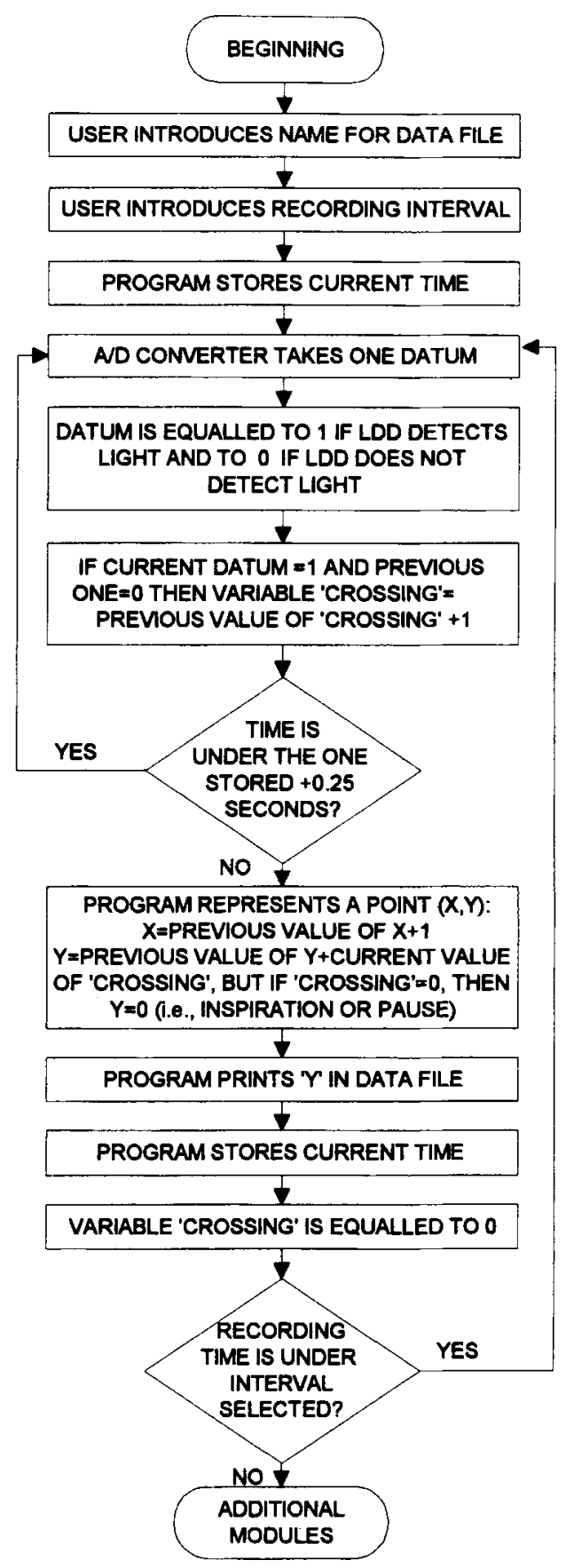

Figure 2. Flux diagram of the basic software that controls the photoelectric-helicoidal spirometer (LDD, light-detecting diode).

justed to a mask (6; Figure 1c) that covers the subject's nostrils and mouth.

This spirometer works as follows: The air that circulates within the interior of the module (5), produces the turbine (1) rotation. As mentioned in the introduction, although this spirometer moves its helix only during exhalation, it provides information about respiratory rate, volume, and flux (see Figure 3 ). The rotation causes the 

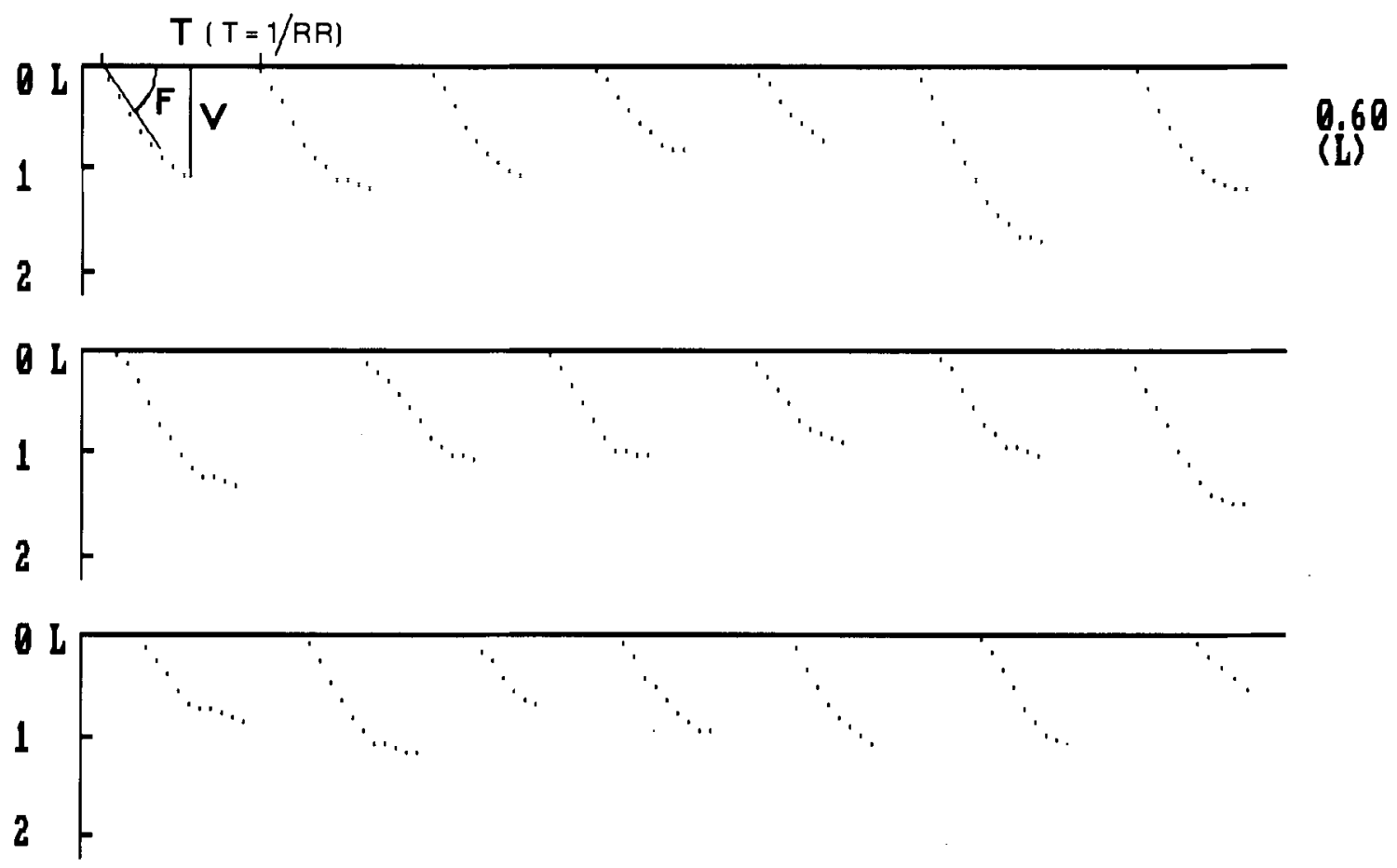

Figure 3. A 90-sec respiratory recording obtained with the photoelectric-helicoidal spirometer. The number at the upper right corner changes constantly, indicating the volume of air exhaled in each moment ( $\mathrm{L}$, liters). The parameters that this device can measure appear in the first respiratory cycle and were drawn later ( $T$, time; $R R$, respiratory rate; $F$, flux; $V$, volume).

opaque plates (2) included in the blades to interrupt the ray of light produced by the LED each time they cross it. The intervals between the four plates allow the ray of light to reach the LDD, so that the interruptions are discrete. By means of the electronic circuit that controls this LED-LDD activity, along with the software described in the next section, the interruptions of the ray of light can be counted and the frequency with which they are produced can also be measured.

This spirometer is calibrated by injecting a known volume of air with a known speed and then counting the number of interruptions of the ray of light originating in the LED. This flux/interruptions ratio is taken into account by the software (next section) in order to determine the exact amplitude of the recordings. However, software enables the user to change this ratio if necessary.

\section{Description of Software}

In this section we describe the basic module of the software that allows the user to record the respiratory activity through the photoelectric-helicoidal spirometer described above. The functions of this basic module are the graphical representation and the storage of respiratory activity. Its flux diagram is shown in Figure 2. More modules can be added to this basic one, but they will not be described here. They are designed to measure certain parameters of the respiratory recordings that are of interest for purposes of interpretation. Specifically, additional modules can measure respiratory rate, tidal volume (both related to the sensorimotor nervous system), and/or air flux (or exhalation slope in which the autonomic nervous system plays an important role).

We were particularly interested in designing a program that could be used with any analog-to-digital (A/D) converter. Since some converters cannot store data, they

Table1

Main Technical Specifications of the Current Version of the Spirometer

\begin{tabular}{lc}
\hline \multicolumn{1}{c}{ Parameter } & Value \\
\hline $\begin{array}{l}\text { Weight of the turbine } \\
\text { Weight of the entire main module } \\
\text { (mask+turbine+LED+LDD+plastic boxes) }\end{array}$ & $0.6 \mathrm{~g}$ \\
$\begin{array}{l}\text { Diameter of the main module (distance LED-LDD) } \\
\text { Air volume detection error (determined by } \\
\text { repetition of the calibration process) }\end{array}$ & $75 \mathrm{gm}$ \\
$\begin{array}{l}\text { Air flux detection error (determined by repetition } \\
\text { of the calibration process) }\end{array}$ & $<0.5 \%$ \\
$\begin{array}{l}\text { Inertia time: average time during turbine continues } \\
\text { its spin after the end of expiration (this parameter } \\
\text { depends on the beginning of the inspiration phase, }\end{array}$ & $\begin{array}{c}0.12 \mathrm{sec} \times \text { air } \\
\text { flux (in liters }\end{array}$ \\
$\quad$ which stops the turbine) & \\
$\begin{array}{l}\text { Temperature range for accurate recordings } \\
\text { Relative-humidity range for accurate recordings }\end{array}$ & $-155^{\circ} \mathrm{C}$ to $45^{\circ} \mathrm{C}$ \\
\hline
\end{tabular}


send each digit to the PC memory before the next one is sampled, and then send the values "one by one" to the computer RAM. With this in mind, we have also used a one-by-one design (described below): each datum sent by the $A / D$ converter is analyzed by the software before the next one is received. Since the speed of this process varies according to the characteristics of each computer, the digitalization rate of the present software depends on real "clock" time. A 1-channel 4-bit converter can use this software, which enables the use of the spirometer even in the simplest PCs (e.g., those using 8088 or 80286 microprocessors). A version for Windows is also available for installation in 80386 or higher computers. The following is a step-by-step explanation of the design.

The basic module begins by asking the user the name of the file in which the recording will be stored and the length, in seconds, of the recording interval. Once the beginning of the recording has been ordered by the user, the program stores the current time, a measure that is essential for marking the sampling rate. Next, the A/D converter digitalizes 1 voltage point from the LED-LDD circuit. The digitalized value is converted into 1 by the program when it reflects the detection of light by the LDD (output voltage of the circuit is not 0 ) and into 0 when it indicates that the LDD has not detected light (voltage $=0$ ). The A/D converter continuously takes data (at the speed enabled by the PC) until current time is under the one stored plus $0.25 \mathrm{sec}$. Thus, at this level, the recorded data consist of alternative chains of $1 \mathrm{~s}$ and $0 \mathrm{~s}$. Each chain of ls is counted as a "crossing" of the helix through the light ray. Therefore, the variable we shall call "crossings" grows by 1 each time the ray is interrupted. In fact, this is the variable that will be represented and stored.

Thus, when current time is equal to the stored time plus $0.25 \mathrm{sec}$, the program advances into a second section, in which data are represented and filed. Figure 3 illustrates the graphic output of the program in a real recording. Each datum represented is defined by an $x$ - (time) coordinate, which is equal to the previous value of $x+1$, and a $y$-coordinate (amplitude), which is equal to the previous value of $y, 0$ this first time, plus the current value of "crossings." Additionally, the current datum is stored in the file whose name was selected by the user at the beginning of the program. What is actually stored is the value of $y$ (though corrected to reflect air volume in liters). Next, the program stores current time again (this will be the new time reference to define the next quarter of a second), variable "crossings" is equaled to 0 , and the program returns to the first section. When the next quarter

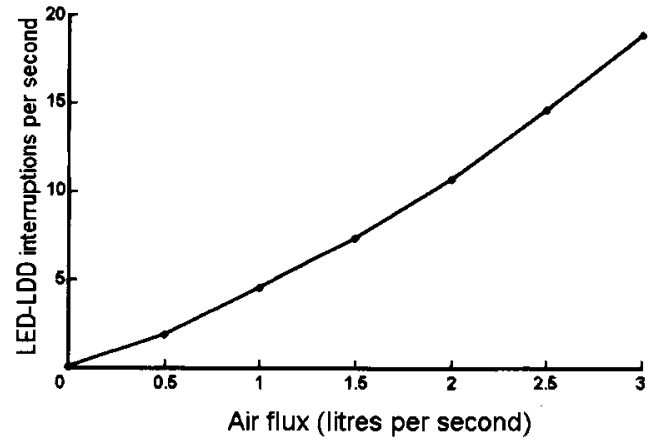

Figure 4. Interaction between the air flux (in liters per second) and the number of LED-LDD interruptions (per second) in the current version of the spirometer $($ LED $=$ light emitter, $L D D=$ light detector). The LED-LDD interruptions were taken during $5 \mathrm{sec}$ for constant air fluxes. The mean value for $1 \mathrm{sec}$ was then calculated.

of a second is finished, the second datum is represented and filed, and so on. This process is repeated until the recording interval chosen by the user has expired.

\section{Equipment Availability}

Table 1 and Figure 4 show the main technical specifications of the current version of the spirometer. This device and its control software and manuals can be obtained by contacting the first author (e-mail: carretie@uam.es). A color, three-dimensional graphical representation of the spirometer can be obtained from our WWW Psychophysiology page (http://www.adi.uam.es/ carretie/ research.htm). The total cost of the equipment, including the secondary or additional software modules (but not the $\mathrm{A} / \mathrm{D}$ converter) is $\$ 345$.

\section{REFERENCES}

Brodal, A. (1981). Neurological anatomy. Oxford: Oxford University Press.

Carretié, L., \& Iglesias, J. (1995). Psicofisiologia: Fundamentos metodológicos [Psychophysiology: Methodological fundamentals]. Madrid: Pirámide.

Kaufman, M. P., \& Schneiderman, N. (1986). Physiological bases of respiratory psychophysiology. In M. G. H. Coles, E. Donchin, \& S. W. Porges (Eds.), Psychophysiology: Systems, processes and applications (pp. 107-121). Amsterdam: Elsevier.

LORIG, T. S., \& SchwarTz, G. (1990). The pulmonary system. In J. T. Cacioppo \& L. G. Tassinary (Eds.), Principles of psychophysiology: Physical, social, and inferential elements (pp. 580-598). Cambridge: Cambridge University Press.

WientJes, C. J. E. (1992). Respiration in psychophysiology: Methods and applications. Biological Psychology, 34, 179-203. 